\title{
Cosmic ray feedback across the sequence of star-forming galaxies
}

\section{Roland M. Crocker ${ }^{a, *}$ and Mark Krumholz ${ }^{a}$}

${ }^{a}$ Research School of Astronomy and Astrophysics, Australian National University, Canberra, Australia E-mail: rcrocker@fastmail.fm, roland.crocker@anu.edu.au

Star formation proceeds inefficiently in galaxies for reasons that remain under debate. In the local ISM it is known that the cosmic rays (CRs) provide a significant fraction of total ISM pressure and therefore contribute to hydrostatic balance. We set out a model for the dynamical effect of CRs, directly accelerated as a result of star formation itself, on the ISM gas column. On the basis of this model, we will explain how CR feed-back sets an ultimate limit to the star formation efficiency of 'ordinary' galaxies (normal spirals and dwarfs). Interestingly, most such galaxies - including the Milky Way - have star formation efficiencies approaching the maximum allowed by cosmic ray feed-back, suggesting they exist in a state of delicate, dynamically-determined equilibrium. However, at the higher surface densities pertinent to star burst systems pionic losses imply that CRs are dynamically unimportant on global scales while, at the same time, guaranteeing that such galaxies are luminous gamma-ray sources. In passing, we explain how our model leads to new insights about the observed $\mathrm{GeV}$ to $\mathrm{TeV}$ spectra of local starbursts.

\footnotetext{
*** 37th International Cosmic Ray Conference (ICRC2021), ***

*** 12-23 July 2021 ***

*** Berlin, Germany - Online ***
}

\footnotetext{
${ }^{*}$ Presenter
} 


\section{Introduction}

In this Proceedings article we report the results of our three recent papers [2, 3, 7] that incorporate a realistic model of cosmic ray (CR) transport through partially-ionised, but largely neutral, star-forming gas in galaxies. Our model for transport incorporates insights developed by $\mathrm{Xu}$, Lazarian and co-workers in their recent studies [8, 12-14] of CR transport in such partiallyionised media. The primary insight that we derive from these studies is that the classical picture of CR diffusion does not work in such gas. Specifically, turbulence that is injected at a large scale by some extrinsic process cannot cascade down to the $\sim 10^{-6} \mathrm{pc}$ gyro-radius scale of the $\sim \mathrm{GeV} \mathrm{CR}$ protons that carry most of the energy density (for reasonable power-law spectral indices of the CR population). There is, thus, an absence of extrinsic turbulence at the gyro-radius scale. This is because of the phenomenon of ion-neutral damping which, for ionisation fractions $\chi \sim 10^{-2}-10^{-4}$ (typical of star-formation-hosting gas: [7]) leads to dissipation of the turbulence at a scale significantly larger than the $\sim \mathrm{GeV}$ gyro-radius scale.

In the absence of extrinsic turbulence, the primary source of magnetic fluctuations at this scale becomes the CRs themselves. These inject magnetic waves via the streaming instability. Such waves, however, are also damped via ion-neutral damping. It turns out that these competing processes then define an equilibrium velocity for CRs moving along the field lines where damping balances streaming losses; to high accuracy, for CRs close to $\sim \mathrm{GeV}$ and for magnetic fields, gas densities, and ionisation fractions typical of star-forming gas, this 'streaming' velocity $v_{s}$ is given by the ion Alfven speed in the medium, $v_{A, i}$ :

$$
v_{s} \simeq v_{A, i} \equiv \frac{\sigma}{\sqrt{2 \chi} M_{A}},
$$

where the parameter $M_{A}$ is known as the Alfvenic Mach number and $\sigma$ is the turbulent velocity dispersion. Note that, given the typical ionisation fraction $\chi$ quoted above, $v_{A, i}$ will be a factor $1 / \sqrt{\chi} \sim(10-100) \times$ faster than the typically-invoked (neutral gas) Alfven velocity, $v_{A}$.

In our work, we also invoke the idea that the dominant magnetic field in such star-forming gas is set up by a turbulent dynamo that raises the magnetic field amplitude close to, but somewhat short of, equipartition with the gas turbulent energy density. We typically expect, therefore, to be operating in a regime where $M_{A}$ is close to but somewhat larger than $1, M_{A} \sim 2$.

In this regime, the dominant, turbulently-generated magnetic field is itself turbulent. Thus, the transport of CRs streaming along field lines (at $\sim v_{A, i}$ ) through star-forming gas can effectively be described via a diffusion coefficient because of the field line random walk. Close to $\sim \mathrm{GeV}$, this diffusion coefficient is well represented by the product of the ion Alfven speed (in full generality, the streaming velocity) and the magnetic field coherence length, which effectively determines the mean free path of the random walk along the field lines. The latter quantity, in the turbulent dynamo scenario, is close to the physical, turbulence injection scale divided by $M_{A}^{3}$. The turbulence injection scale is itself close to the scale height of the gas column, $z_{*}$

$$
z_{*} \equiv \frac{\sigma^{2}}{g_{*}},
$$

(in which the turbulent velocity dispersion of the gas $\sigma$ is assumed constant) and

$$
g_{*}=2 \pi G \frac{\Sigma_{\mathrm{gas}}}{f_{\mathrm{gas}}}
$$




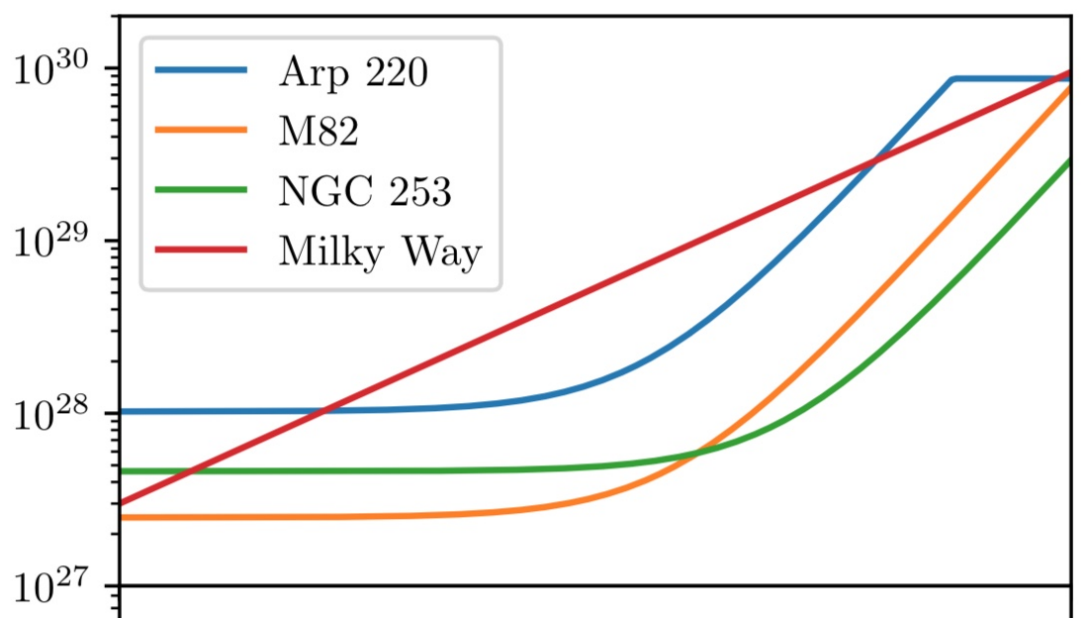

Figure 1: CR diffusion coefficient $\kappa$ as a function of CR energy as computed for the listed starburst galaxies in [7] and reproduced from there. The Milky Way line uses an empirical estimate of the diffusion coefficient together with the measured gas surface density and scale height. The kink in the lines for Arp 220 marks to the energy for which the CR streaming speed approaches c.

defines the reference gravitational acceleration for the planar distribution of gas with surface density $\Sigma_{\text {gas }}$ and where $f_{\text {gas }} \equiv \Sigma_{\text {gas }} / \Sigma_{\text {tot }}$, where $\Sigma_{\text {tot }}$ includes both stars and gas.

We therefore have that the magnetic field coherence length is around

$$
l_{\mathrm{coh}, \mathrm{B}} \simeq \frac{z_{*}}{M_{A}^{3}},
$$

and the resultant heuristic diffusion coefficient for CR transport in the partially-ionised gas:

$$
\kappa \simeq v_{A, i} \frac{l_{\mathrm{coh}, \mathrm{B}}}{3} .
$$

Note that while the above approximation works well for $E_{\mathrm{CR}} \sim \mathrm{GeV}$, for typical ISM environments it starts to break down in the $\mathrm{TeV}$ range because of the progressive decline of the number density of CRs above a given energy (and thus able to excite, via the streaming instability, magnetic waves at the corresponding gyro-radius scale, which itself grows linearly with energy in the relevant relativistic regime). Effectively, because of this decline, the equilibrium speed of the streaming where damping saturates the streaming instability - increases, only ultimately bound by the speed of light. The result of this behaviour is that the diffusion coefficient is quasi energy-independent in the $\sim \mathrm{GeV}$ range, but then increases with energy (as the streaming speed diverges from $v_{A, i}$ ); cf. fig. 1.

All this has interesting consequences for the predicted $\gamma$-ray phenomenology of star-forming galaxies for the (expected) case that a galaxy's overall $\gamma$-ray spectrum is dominated by collisions between hadronic cosmic rays and dense gas. In particular, we expect (given the mild energyindependence of the $p p$ cross-section well above the threshold) that the $\sim \mathrm{GeV} \gamma$-ray spectrum is a featureless power law reflecting the distribution of the parent protons, but that this spectrum then steepens into the $\mathrm{TeV} \gamma$-ray regime where the parent protons and heavier ions start to diffuse more quickly out of the gas column into the regime where $v_{s} \gg v_{A, i}$. With few free parameters, we have shown quantitatively that such behaviour well reproduces the observed spectra of a number of nearby, $\gamma$-ray detected galaxies; cf. fig. 2, which is reproduced from [7]. 


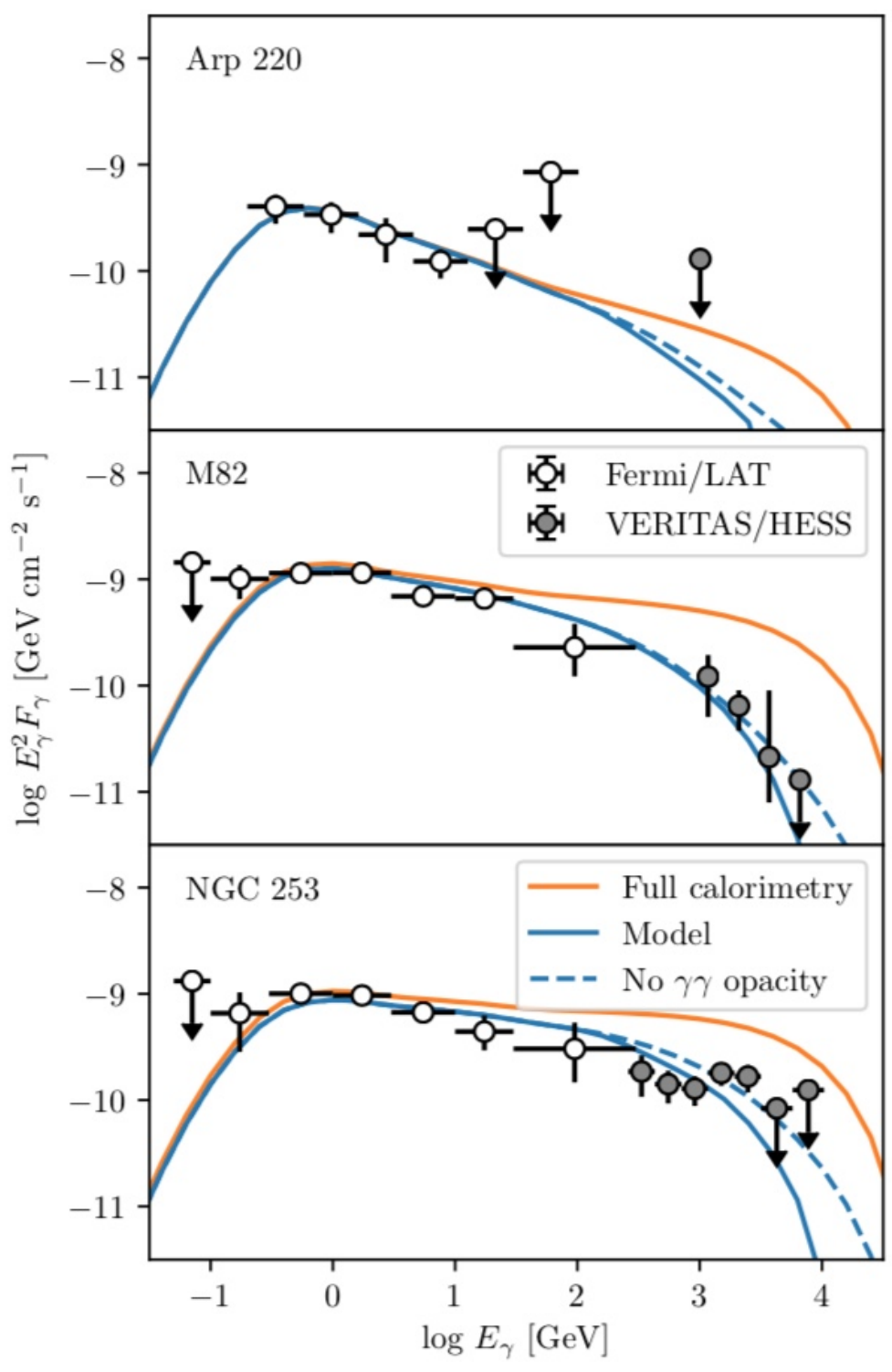

Figure 2: Spectra of NGC 253, M82, and Arp 220, computed as described in [7] and reproduced from there. The solid blue lines show the standard model, circles with error bars show observations. Open circles are from the Fermi/LAT 8-year catalog [1] for NGC 253 and M82, and from the anlaysis of Fermi data by Peng et al. [9] for Arp 220. Grey filled circles are from VERITAS for M82 [11] and Arp 220 [4], and from HESS for NGC 253 [5]. Horizontal error bars show the energy band over which a particular observation is made, while vertical error bars show $1 \sigma$ uncertainties; points with downward arrows indicate $1 \sigma$ upper limits for bands without detections.

Given that this phenomenological match serves as a confirmation that our approach to CR transport is credible, we have subsequently investigated the potential dynamical effects of CRs in star-forming galaxies, in particular, the effect of CRs on star-forming gas. We know that, in the local disk of the Milky Way, CRs have energy density comparable to other components of the ISM. This means that they provide a significant part of the total pressure that supports the gas column in hydrostatic equilibrium. But one can ask the question: what would happen as the CR pressure were dialled up and up? Is there a maximum allowed flux of CRs that can diffuse through the gas column beyond which the gas column loses hydrostatic equilibrium or is otherwise rendered unstable? In other words, what is the analogue of the Eddington limit, but for CRs [10]?

This question can be tackled quantitatively via a relatively simple semi-analytic treatment $[2,3]$ 
which involves determining the parameter space over which we can find numerical solutions for two coupled ordinary differential equations that simultaneously specify CR transport and hydrostatic equilibrium in terms of four dimensionless functions, $s(\xi), r(\xi)=d s / d \xi, p_{c}(\xi)$, and $\mathcal{F}_{c}(\xi)$, which represent the dimensionless versions of the gas column, gas density, CR pressure, and CR flux as a function of dimensionless height $\xi$

$$
\xi \equiv \frac{z}{z_{*}}
$$

The final form of the dimensionless cosmic ray transport equation is:

$$
\frac{d}{d \xi}\left[-\left(\frac{d s}{d \xi}\right)^{-q} \frac{d p_{c}}{d \xi}\right]=-\tau_{\mathrm{abs}} \frac{d s}{d \xi} p_{c}+\tau_{\mathrm{stream}} \frac{d p_{c}}{d \xi}
$$

where

$$
\begin{aligned}
\tau_{\text {stream }} & =\frac{\beta_{s}}{K_{*} \beta}=\frac{1}{K_{*}} \frac{v_{s}}{\sigma} \\
\tau_{\text {abs }} & =\frac{\tau_{\mathrm{pp}}}{K_{*} \beta},
\end{aligned}
$$

in which $K_{*}$ is a normalising diffusion coefficient, and $\beta_{s}$ and $\beta$ are the streaming velocity and turbulent velocity dispersion in units of $\mathrm{c}$. Equation 7 states that the change in CR flux with respect to height (the LHS) is equal to the rate at which CRs are lost due to collisions (the first term on the RHS) and dissipation of CR energy into Alfvén waves, and ultimately into thermal energy, via the streaming instability (the second term on the RHS). The two crucial parameters $\tau_{\text {abs }}$ and $\tau_{\text {stream }}$ represent the 'absorption' and 'streaming' optical depths of the gas column to CRs. The final form of the complementary ODE expressing hydrostatic balance is:

$$
\frac{d p_{c}}{d \xi}+\phi_{\mathrm{B}} \frac{d^{2} s}{d \xi^{2}}=-\left(1-f_{\mathrm{gas}}\right) \frac{d s}{d \xi}-f_{\mathrm{gas}} s \frac{d s}{d \xi} .
$$

The terms here are, from left to right, the pressure gradient due to CRs, the pressure gradient due to turbulence ( $\phi_{B} \sim 1$ is a parameter quantifying additional magnetic support), the gravitational acceleration due to stellar gravity, and the acceleration due to gas self-gravity.

Finally, our system of Equation 7 and Equation 10 is fourth order, thus requiring four boundary conditions. These are fully described in $[2,3]$ but it is important here to describe the $\mathrm{BC}$ corresponding to the CR flux at $\xi=0$ :

$$
-\left.\left(\frac{d s}{d \xi}\right)^{-q} \frac{d p_{c}}{d \xi}\right|_{\xi=0}=\frac{\tau_{\text {stream }}}{\beta_{s}} \frac{F_{c, 0}}{F_{*}} \equiv f_{\text {Edd }},
$$

where the quantity $f_{\text {Edd }}$ is the ratio of the incoming CR flux to the Eddington flux, defined here as the flux for which the momentum flux carried in the $+z$ direction by the cosmic rays leaving the lower boundary $\left(F_{c, 0}\right)$ matches the momentum flux in the $-z$ direction due to gravity:

$$
F_{*}=\frac{\pi G c}{f_{\mathrm{gas}}} \Sigma_{\mathrm{gas}}^{2}
$$

Note here that $F_{c, 0}$ is enhanced by the factor $\tau_{\text {stream }} / \beta_{s}$ that accounts for the diffusive nature of the CR transport (c.f.[10]). 

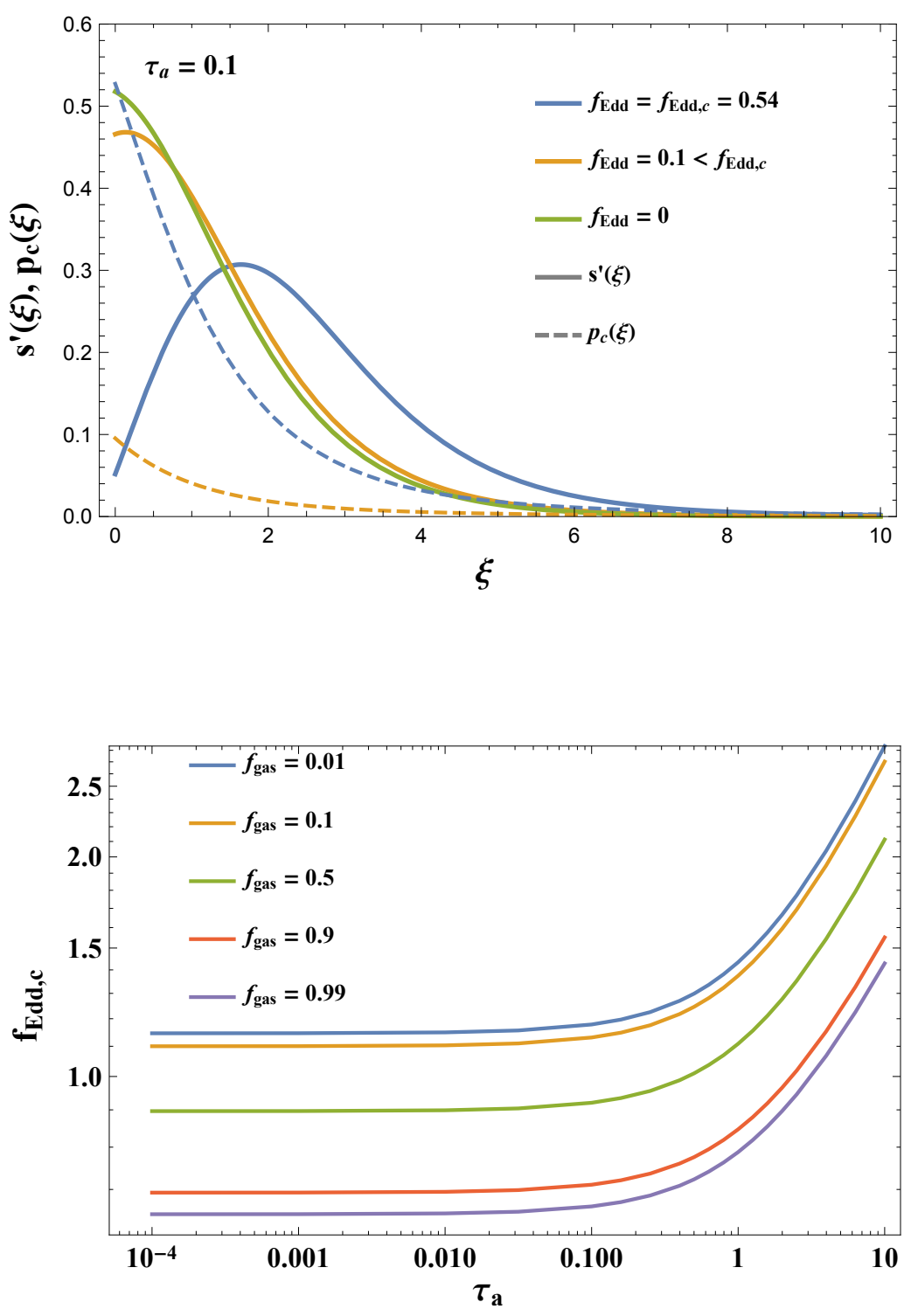

Figure 3: Profiles of (dimensionless) volumetric density $r(\xi)=s^{\prime}(\xi)$ (solid) and (dimensionless) CR pressure $p_{c}(\xi)$ (dashed) for various representative cases. This figure is for $\tau_{\mathrm{abs}}=0.1, q=1 / 4, \tau_{\text {stream }}=$ $1, f_{\text {gas }}=0.9$. The blue curves are evaluated for the critical $f_{\text {Edd }}$ case and the yellow curves are for a subcritical $f_{\text {Edd }}$ value; the solid green curve is the density profile of a gas column supported purely by turbulence. Plot reproduced from [3].

Figure 4: CR Eddington limit $f_{\text {Edd,c }}$ as a function of effective optical depth $\tau_{\text {abs }}$. $f_{\text {Edd,c }}$ versus $\tau_{\text {abs }}$ at fixed $\tau_{\text {stream }}=1$ and for a range of gas fractions $f_{\text {gas }}$ as indicated in the legend; a higher $f_{\text {gas }}$ renders the column (somewhat) less stable (i.e., reduces $f_{\text {Edd,c }}$ ), while a higher $\tau_{\mathrm{abs}}$ renders the column more stable once we are in the optically thick regime (because hadronic collisions reduce the steady state $\mathrm{CR}$ pressure). Plot reproduced from [3].

We must solve Equation 7 and Equation 10 numerically. Because the boundary conditions for the system are specified at different locations, the system forms a boundary value problem, which we solve using a shooting algorithm.

A crucial feature of solutions to this system is that, as $f_{\text {Edd }}$ increases at fixed $\tau_{\text {abs }}$ and $\tau_{\text {stream }}$, the dimensionless midplane density $s^{\prime}(0)$ decreases monotonically, approaching zero at a finite value of $f_{\text {Edd }}$. We illustrate this behaviour in Figure 3. We refer to the value of $f_{\text {Edd }}$ for which this occurs as the critical Eddingtion ratio, $f_{\text {Edd,c }}$. No solutions exists for $f_{\text {Edd }}>f_{\text {Edd,c }}$, and thus $f_{\text {Edd,c }}$ represents the largest Eddington ratio for which it is possible for a gas column through which CRs are forced to remain in equilibrium. Larger values of $f_{\text {Edd }}$ necessarily render the system unstable.

The final step in our calculation is to translate our stability limit from dimensionless $\left(f_{\text {Edd }}\right.$, 


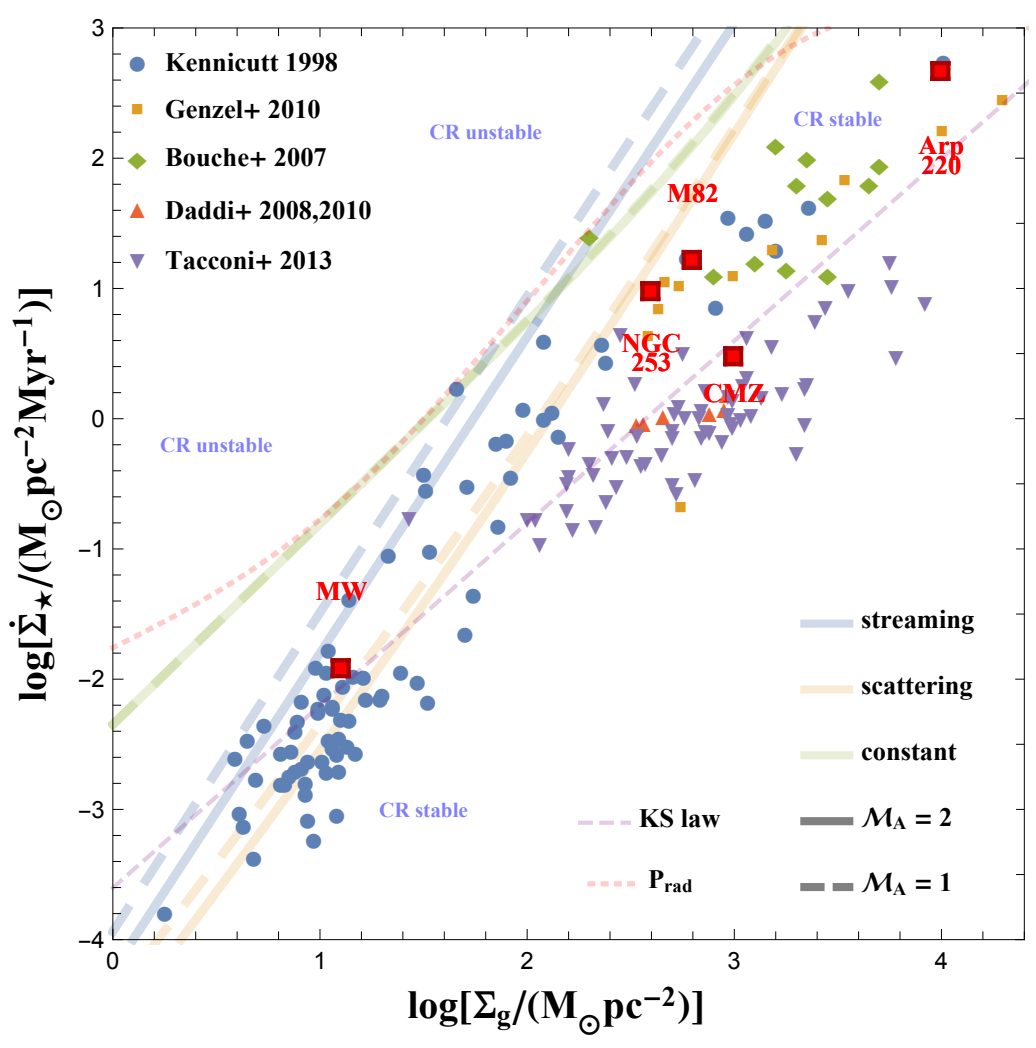

Figure 5: Thick coloured lines show critical stability curves $\dot{\Sigma}_{\star, c}$, i.e., the star formation rate per unit area at which $\mathrm{CR}$ pressure precludes hydrostatic equilibrium, computed assuming one of three different CR transport modes as indicated (with streaming constituting our fiducial model), and for $\mathcal{M}_{A}=2$ (solid) and $\mathcal{M}_{A}=1$ (dashed) together with fiducial parameter choices for all models, and using the fits for $f_{\text {gas }}, \chi$, and $\sigma$ as a function of $\Sigma_{\text {gas }}$ given in [3]. The dashed, diagonal, purple line is the Kennicutt [6] star formation scaling. Finally, points show observations drawn from the sources nominated in the caption (see [3], from where this figure is reproduced, for full references).

$\left.\tau_{\text {abs }}, \tau_{\text {stream }}\right)$ to the physical variables describing a star-forming galactic disc. In particular, we are interested in the highest star formation rate (since star formation produces supernovae that are the primary source of CRs) that a disk can sustain before it becomes unstable to the development of CR-driven outflows. Full details of how to perform the conversion from dimensionless into physical units are presented in [3], but are straightforward. We show, effectively, the translation of the dimensionless critical stability curve (à la Figure 4) into the physical parameter space of star formation rate surface density vs. gas surface density - i.e., the Kennicutt-Schmidt plane - in Figure 4 where we draw the reader's attention to the light blue 'streaming' curves in particular.

This figure demonstrates something quite remarkable: the CR stability curve patrols a region very close to the top of the occupied part of the $\left(\Sigma_{\text {gas }}, \dot{\Sigma}_{\star}\right)$ plane for star-forming galaxies with low gas surface densities typical of the Galaxy and local dwarfs. This correspondence strongly suggests that CR feedback on the neutral gas is an important mechanism in such galaxies: it either limits the ability of galaxies to make excursions above the locus where most of them lie, or it might be responsible for launching winds and ejecting gas in galaxies that do wander upwards to higher star formation rates.

Conversely, it is evident that, at the higher gas surface densities encountered in local starbursts and high-redshift star-forming galaxies, all the critical curves (not just the one for our fiducial model) diverge away from the observed distribution of galaxies. This implies that CRs cannot drive 
winds in these systems [2]. The very process of hadronic collisions that renders these systems $\gamma$-ray bright, is what guarantees that CR do not play an important dynamical role in them.

\section{References}

[1] Abdollahi, S., Acero, F., Ackermann, M., et al. 2020, ApJS, 247, 33. doi:10.3847/15384365/ab6bcb

[2] Crocker, R. M., Krumholz, M. R., \& Thompson, T. A. 2021, MNRAS, 502, 1312. doi:10.1093/mnras/stab148

[3] Crocker, R. M., Krumholz, M. R., \& Thompson, T. A. 2021, MNRAS, 503, 2651. doi:10.1093/mnras/stab502

[4] Fleischhack, H. \& VERITAS Collaboration 2015, 34th International Cosmic Ray Conference (ICRC2015), 34, 745

[5] H. E. S. S. Collaboration, Abdalla, H., Aharonian, F., et al. 2018, A\&A, 617, A73. doi:10.1051/0004-6361/201833202

[6] Kennicutt, R. C. 1998, ApJ, 498, 541. doi:10.1086/305588

[7] Krumholz, M. R., Crocker, R. M., Xu, S., et al. 2020, MNRAS, 493, 2817. doi:10.1093/mnras/staa493

[8] Lazarian, A. 2016, ApJ, 833, 131. doi:10.3847/1538-4357/833/2/131

[9] Peng, F.-K., Wang, X.-Y., Liu, R.-Y., et al. 2016, ApJ, 821, L20. doi:10.3847/2041$8205 / 821 / 2 / \mathrm{L} 20$

[10] Socrates, A., Davis, S. W., \& Ramirez-Ruiz, E. 2008, ApJ, 687, 202. doi:10.1086/590046

[11] VERITAS Collaboration, Acciari, V. A., Aliu, E., et al. 2009, Nature, 462, 770. doi:10.1038/nature08557

[12] Xu, S., Yan, H., \& Lazarian, A. 2016, ApJ, 826, 166. doi:10.3847/0004-637X/826/2/166

[13] Xu, S. \& Lazarian, A. 2017, New Journal of Physics, 19, 065005. doi:10.1088/13672630/aa6ec 9

[14] Yan, H. \& Lazarian, A. 2008, ApJ, 673, 942. doi:10.1086/524771 